\title{
Dietary intake and metabolic profile of patients with Barrett's oesophagus
}

\author{
L. K. Herlihy ${ }^{1,2}$, L. A. Healy ${ }^{1,3}$, J. V. Reynolds ${ }^{1}$ and F. E. Lithander ${ }^{1}$ \\ ${ }^{1}$ School of Medicine, University of Dublin, Trinity College, Dublin, Ireland, ${ }^{2}$ School of Biological Sciences, Dublin Institute \\ of Technology, Dublin, Ireland and ${ }^{3}$ Department of Clinical Nutrition, St James's Hospital, Dublin, Ireland
}

Barrett's oesophagus is a pre-malignant condition that develops in response to chronic exposure of the oesophageal epithelium to the acid and bile contents of gastro-oesophageal reflux ${ }^{(1)}$. Abdominal obesity and poor dietary and nutrient intake are risk factors for Barrett's oesophagus $^{(2)}$. The aim of the current cross-sectional study was to assess dietary intake, anthropometry and metabolic profile in patients with Barrett's oesophagus.

Male patients ( $n$ 23) with histologically confirmed Barrett's oesophagus were recruited from the Barrett's Oesophagus Clinic at St James's Hospital, Dublin, Ireland. Height, weight, waist circumference and blood pressure were measured. Segmental bioelectrical impedance was analysed (Tanita UK Ltd, Middlesex, UK), and fasting blood samples (lipid profile and glucose) were taken. Each patient completed a $3 \mathrm{~d}$ dietary record. Dietary intake data were analysed using nutrient analysis software (WISP for Windows Version 3, Tinuviel Software, Anglesey, UK) and subsequent statistical analysis was performed using SPSS Version 16.0 for Windows (SPSS Inc., Chicago, IL, USA).

The mean (SD) age was 54 (12) years, BMI was $28.6(4.2) \mathrm{kg} / \mathrm{m}^{2}$, waist circumference was 104.2 (10.5) cm, blood pressure 149 (21)/95 (17) $\mathrm{mmHg}$ and serum cholesterol was $5.16(1.10) \mathrm{mmol} / \mathrm{l}$. Data on Barrett's oesophagus segment length were available for 20 patients, $n=13$ had long-segment $(\geq 3 \mathrm{~cm})$ and $n=7$ had short-segment Barrett's $(<3 \mathrm{~cm})$. Mean (sD) dietary energy intake was 9.1 (2.3) MJ/d; protein accounted for 16.9 (2.6) \% energy (\%en), fat for 35.1 (6) \%en, carbohydrate for 44.9 (7.6) \%en and alcohol for 7.1 (8.4) \%en. When classified as per $\mathrm{BMI}^{(3)}, n=4$ patients were of healthy weight, $n=10$ were overweight and $n=9$ were obese. There were no differences in anthropometric measurements between patients with long-segment or short-segment Barrett's. Patients with long-segment Barrett's had higher intakes of energy than those with short-segment Barrett's (10.0 (2.2) v. 7.2 (1.4) MJ/d, $P=0.009)$. Negative associations were observed between systolic blood pressure and dietary PUFA $(r=-0.69, P=0.006)$ and fish consumption $(r=-0.60$, $P=0.023)$. Eight patients $(38 \%)$ met the criteria for the metabolic syndrome ${ }^{(4)}$.

In summary, the prevalence of central obesity, hypertension and the metabolic syndrome within this cohort exceed that of the general Irish adult population ${ }^{(5)}$. Patients with Barrett's oesophagus may be at an increased risk of CVD and type-2 diabetes mellitus in addition to oesophageal adenocarcinoma. These data highlight the need for dietary and lifestyle intervention to decrease the risk of co-morbidities within this patient group.

1. Jankowski JA, Harrison RF, Perry I et al. (2000) Barrett's metaplasia. Lancet 356, 2079-2085.

2. Edelstein ZR, Farrow DC, Bronner MP et al. (2007) Central adiposity and risk of Barrett's esophagus. Gastroenterology 133, $403-411$.

3. World Health Organisation (WHO) (1998) Obesity: Preventing and Managing the Global Epidemic. Report of a WHO consultation on Obesity, Geneva, 3-5 June 1997. Geneva: WHO.

4. International Diabetes Federation (IDF) (2005) The IDF Consensus Worldwide Definition of the Metabolic Syndrome. http://www.idf.org/webdata/docs/ MetS_def_update2006.pdf

5. Waterhouse DF, McLaughlin AM, Sheehan F et al. (2009) An examination of the prevalence of the IDF- and ATPIII-defined metabolic syndrome in an Irish screening population. Ir J Med Sci 178, 161-166. 